\title{
Geometría y Dinámica del oscilador armónico 2-dimensional
}

\author{
Misael Avendaño-Camacho \\ Departamento de Matemáticas \\ Universidad de Sonora \\ e-mail: misaelave@gmail.com
}

\begin{abstract}
Resumen
En este artículo se estudia la geometría y la dinámica del oscilador armónico 2-dimensional como un sistema de ecuaciones diferenciales lineales en $\mathbb{R}^{4}$. Se describen explícitamente los conjuntos invariantes del oscilador los cuales en su gran mayoría resultan ser toros 2-dimensionales. Luego, se estudia la dinámica del sistema sobre los toros de Liouville y se exhibe la gran dependencia cualitativa que esta tiene de una relación aritmética entre sus frecuencias, pasando de tener órbitas periódicas en los toros a tener trayectorias densas sobre éstos. Si bien este hecho es bien conocido en la teoría de sistemas Hamiltonianos integrables, aqui presentamos los resultados haciendo solamente uso de conceptos básicos de cálculo y ecuaciones diferenciales.
\end{abstract}

\section{Introducción}

El oscilador armónico $n$-dimensional o con $n$ grados de libertad es un modelo bien conocido y elemental tanto en física como en matemáticas. Por ejemplo, aparece como el modelo lineal en el estudio de oscilaciones, que es un área de interés común en ambas disciplinas. También lo podemos encontrar en mecánica clásica y cuántica, ya que es el modelo más elemental posible de un sistema Hamiltoniano [1, 5, 7]. En matemáticas, el oscilador armónico aparece a menudo en los cursos de cálculo y ecuaciones diferenciales; pues es uno de los ejemplos básicos que aparecen en el estudio de ecuaciones diferenciales lineales de segundo orden y/o sistemas de ecuaciones diferenciales lineales de primer orden [8]. Sin embargo, muchos de los aspectos geométricos que pueden ser realmente interesantes y más o menos simples de explicar suelen pasar inadvertidos o rara vez son discutidos en cursos o textos elementales de ecuaciones diferenciales. De hecho, el oscilador armónico tiene una gran relación con importantes tópicos relevantes en matemáticas como la fibración de Hopf, los flujos ergódicos en el toro y la topología de la 3-esfera, por mencionar algunos [10].

El propósito de este artículo es estudiar la geometría y la dinámica del oscilador armónico 2-dimensional visto como un sistema de ecuaciones diferenciales lineales en $\mathbb{R}_{(q, p)}^{4}$. Específicamente, se describen los conjuntos invariantes del oscilador armónico, que en su mayoría resultan ser toros 2-dimensionales, y la dinámica del sistema sobre ellos. El oscilador armónico 2-dimensional resulta ser un sistema Hamiltoniano con dos grados de libertad con Hamiltoniano dado por $H\left(q_{1}, q_{2}, p_{1}, p_{1}\right)=\frac{1}{2}\left(p_{1}^{2}+p_{2}^{2}\right)+\frac{1}{2}\left(\omega_{1}^{2} q_{1}+\omega_{2}^{2} q_{2}\right)$, donde $\omega_{1}, \omega_{2}$ son constantes reales que usualmente son llamadas frecuencias del oscilador. Un detalle interesante, al menos desde el punto del vista del autor, es que la dinámica sobre los toros invariantes resulta muy sencilla (lineal), pero altamente sensible a la relación aritmética entre sus frecuencias. 
Si $\frac{\omega_{1}}{\omega_{2}}$ es un número racional, los toros invariantes están foliados por órbitas periódicas del oscilador. En cambio, si $\frac{\omega_{1}}{\omega_{2}}$ es irracional las órbitas del oscilador sobre los toros invariantes ya no son más periódicas, sino que ahora cada órbita llena densamente algún toro invariante. Este hecho es bastante conocido y elemental entre los especialistas en sistemas Hamiltonianos integrables [1,3]. En este sentido, nada de lo presentado aquí es nuevo y existe una amplia literatura en el tema, los textos [1, 2, 3, 9] son una muestra de ello. Lo relevante de este artículo está en la forma en que se presentan y demuestran los resultados, pues la simpleza del oscilador armónico 2-dimensional permite desarrollar la teoría haciendo uso sólo de nociones elementales de cálculo de varias variables y ecuaciones diferenciales; lo que lo hace accesible para una audiencia bastante amplia para quienes está dirigido este artículo. El propósito subyacente en este trabajo es que el lector descubra la riqueza de los conceptos matemáticos que se pueden encontrar en un modelo tan sencillo como el oscilador armónico 2-dimensional.

El presente artículo está organizado de la siguiente forma. En la sección 2 se presenta una breve introducción a la teoría de sistemas Hamiltonianos. Puesto que a lo largo de este trabajo se hace referencia al hecho de que el oscilador armónico es un sistema Hamiltoniano, esta sección está dirigida a aquellos que no tengan experiencia alguna con la teoría de sistemas Hamitonianos. A manera de preámbulo, en la sección 3 se presenta la geometría del oscilador armónico 1-dimensional desde el punto de vista de un sistema dinámico, haciendo énfasis en el uso e interpretación de las coordenadas acción-ángulo. En la sección 4 se introduce el oscilador armónico 2-dimensional y se analiza principalmente los aspectos dinámicos de este sistema. En la sección 5 se presenta la geometría del oscilador armónico 2-dimensional, es decir, se describen analíticamente los conjuntos invariantes de este sistema en su espacio fase mostrando que casi en su totalidad está foliado por toros 2-dimensionales invariantes. La sección 6 presenta un análisis detallado de la dinámica del oscilador armónico sobre los toros invariantes exhibiendo la dependencia que hay entre la dinámica del sistema y la relación entre las frecuencias.

\section{Nociones básicas de sistemas Hamiltonianos en $\mathbb{R}^{2 n}$.}

Consideremos el espacio Euclideano $\mathbb{R}^{2 n}:=\left\{\left(q_{1}, q_{2}, \cdots, q_{n}, p_{1}, p_{2}, \cdots, p_{n}\right) \mid p_{i}, q_{i} \in \mathbb{R} i=\right.$ $1,2, \ldots, n\}$. Sea $H: \mathbb{R}^{2 n} \rightarrow \mathbb{R}$ una función suave. Un sistema Hamiltoniano (autónomo) con $n$-grados de libertad es un sistema de $2 n$ ecuaciones diferenciales ordinarias de la forma:

$$
\begin{aligned}
\frac{d q_{i}}{d t} & =\frac{\partial H}{\partial p_{i}}(q, p), \\
\frac{d p_{i}}{d t} & =-\frac{\partial H}{\partial q_{i}}(q, p) .
\end{aligned}
$$

con $i=1, \ldots, n$. A la función $H$ se le llama el Hamiltoniano del sistema (1). El campo vectorial $X_{H}$ definido por el sistema (1) es llamado campo Hamiltoniano asociado a $H$. Explícitamente, este campo tiene la forma

$$
X_{H}=\left(\frac{\partial H}{\partial p}(q, p),-\frac{\partial H}{\partial q}(q, p)\right) \text {. }
$$


En mecánica clásica, el espacio Euclideano $\mathbb{R}_{(q, p)}^{2 n}$ es llamado el espacio fase del sistema, el espacio $\mathbb{R}_{q}^{n}$ se conoce como espacio de configuración y los vectores $p=\left(p_{1}, p_{2}, \ldots, p_{n}\right)$ y $q=\left(q_{1}, q_{2}, \ldots, q_{n}\right)$ son llamados vector momento y vector de posición, respectivamente.

La principal característica de un sistema Hamiltoniano es que la función Hamiltoniana $H$ es siempre una integral primera del sistema. Recuérdese que una integral primera de un sistema de ecuaciones autónomo (ó campo vectorial) es una función que es constante a lo largo de las trayectorias del sistema, [1, 2]. Geométricamente, esto significa que las soluciones de un sistema Hamiltoniano están contenidas en los conjuntos de nivel de la función $H$. En mecánica, suelen aparecer sistemas Hamiltonianos definidos por funciones de forma $H(q, p)=T(p)+U(q)$, donde $T$ y $U$ representan la energía cinética y potencial del sistema, respectivamente. Por lo que la función $H$ se interpreta como la energía total del sistema. En este contexto, la propiedad de que el Hamiltoniano es una integral primera del sistema suele interpretarse como el principio de conservación de energía.

Ejemplo 2.1 El movimiento de una partícula en el espacio cuya masa es $m>0$ y que se mueve en un campo potencial $V(q), q \in \mathbb{R}^{3}$, es descrito por la segunda ley de Newton

$$
m \ddot{q}_{i}=-\frac{\partial V}{\partial q_{i}}, \quad\left(i=1,2,3, \dot{q}_{i}=\frac{d q_{i}}{d t}\right) .
$$

Ahora, definiendo el vector de momento como $p=m \dot{q}, q \in \mathbb{R}^{3}$, el sistema anterior es equivalente al siguiente sistema lineal en el plano $(p, q)$ dado por

$$
\begin{aligned}
\dot{q} & =\frac{1}{m} p, \\
\dot{p} & =-\frac{\partial V}{\partial q_{i}} .
\end{aligned}
$$

$Y$ éste último es un sistema Hamiltoniano determinado por el función Hamiltoniana

$$
H(p, q)=\sum_{i=1}^{3} \frac{1}{2 m} p_{i}^{2}+V(q)
$$

\section{Oscilador armónico 1-dimensional}

El oscilador armónico 1-dimensional es el sistema dinámico en $\mathbb{R}$ determinado por la ecuación diferencial de segundo orden

$$
\ddot{q}+\omega^{2} q=0, \quad q \in \mathbb{R},
$$

donde $\omega$ es una constante real positiva. Si introducimos la variable $p=\dot{q}$, la ecuación ecuación

(2) se transforma en el siguiente sistema de ecuaciones diferenciales lineales de primer orden:

$$
\begin{aligned}
& \dot{q}=p, \\
& \dot{p}=-\omega^{2} q .
\end{aligned}
$$


El sistema (3) es Hamiltoniano en $\mathbb{R}^{2}$ con función Hamiltoniana

$$
H(q, p)=\frac{1}{2}\left(p^{2}+\omega^{2} q^{2}\right)
$$

En mecánica, el oscilador armónico 1-dimensional (3) modela el movimiento de una masa $m$ colgada de un resorte en el que se desprecia cualquier fuerza de fricción (ver figura 1).

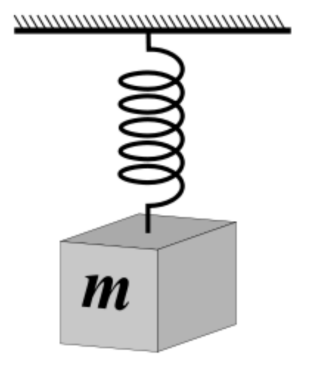

Figura 1: Sistema físico modelado por el oscilador armónico.

En este contexto, la variable $q$ representa el desplazamiento (vertical) de la masa con respecto a su posición de equilibrio y la variable $p$ representa el momento de la masa. Es claro que el movimiento de la masa sujeta al resorte resulta ser periódico, pues estamos despreciando cualquier efecto de fricción. En este caso, $\omega$ representa la frecuencia del movimiento.

Analíticamente, la trayectoria $\alpha(t)=(q(t), p(t)$ del sistema (3) que pasa por el punto $\left(q_{0}, p_{0}\right)$ al tiempo $t_{0}=0$ está dada por

$$
\left(\begin{array}{l}
q(t) \\
p(t)
\end{array}\right)=\left(\begin{array}{rr}
\cos \omega t & \frac{1}{\omega} \operatorname{sen} \omega t \\
-\omega \operatorname{sen} \omega t & \cos \omega t
\end{array}\right)\left(\begin{array}{l}
q_{0} \\
p_{0}
\end{array}\right) .
$$

De aquí se sigue que las órbitas del sistema son períodicas, con periodo $T=\frac{2 \pi}{\omega}$ y por lo tanto las trayectorias del sistema son curvas cerradas. Como la función Hamiltoniana (4) es una integral primera del oscilador armónico 1-dimensional (3), las trayectorias del sistema están contenidas en los conjuntos de nivel de $H$. Por otro lado, para cada número real positivo $E>0$, el conjunto de nivel $S_{E}=\left\{(q, p) \mid p^{2}+\omega^{2} q^{2}=2 E\right\}$ de (4) es una curva regular cerrada, una elipse en particular. En consecuencia, las trayectorias del oscilador armónico 1-dimensional son exactamente las elipses que describen los conjuntos de nivel regulares de la función $H$, (ver figura 2). En esta situación, podemos introducir una sistema de coordenadas especiales $(I, \varphi) \in \mathbb{R}^{+} \times \mathbb{S}^{1}$, llamadas coordenadas acción-ángulo . Las coordenadas $(I, \varphi)$ se definen de la siguiente forma: para cada $E>0$ fijo, denotemos por $\mathcal{D}(E)$ el dominio en $\mathbb{R}^{2}$ acotado por la trayectoria $S_{E}$, la cual es una elipse. Sea $A(E)=\oint_{S_{E}} p d q$, el área del dominio $\mathcal{D}(E)$. Entonces, el periodo $T(E)$ de la curva $S_{E}$ está dado por $T(E)=\frac{\partial A}{\partial E}$. Por último, las coordenadas acción-ángulo se definen por

$$
I=\frac{1}{2 \pi} A(E), \quad \varphi=\frac{2 \pi t}{T(E)} .
$$




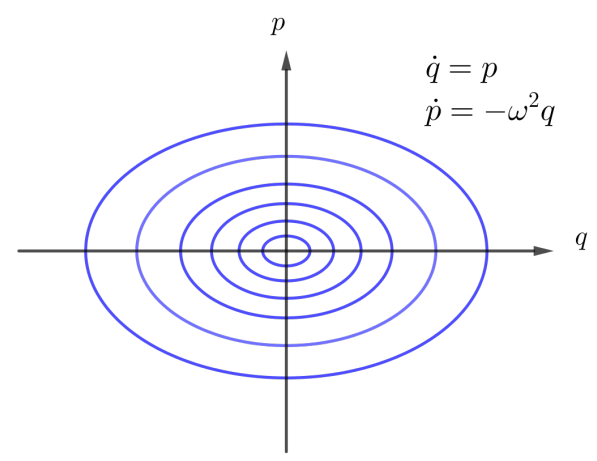

Figura 2: Trayectorias del Oscilador Armónico 1-dimensional.

Usando las expresiones en (6) y el flujo del oscilador armónico (5), se deriva el cambio de coordenadas entre el sistema cartesiano $(q, p)$ y las coordenas accion-angulo $(I, \varphi)$ el cual es dado por

$$
q=\sqrt{\frac{2 I}{\omega}} \cos \varphi, \quad p=-\sqrt{2 \omega I} \operatorname{sen} \varphi .
$$

Bajo este cambio de coordenadas, el oscilador armónico 1-dimensional (3) se transforma en

$$
\begin{aligned}
\dot{I} & =0, \\
\dot{\varphi} & =\omega,
\end{aligned}
$$

Notemos que este sistema es bastante simple y es es fácilmente integrable por cuadraturas. Más aún, el cambio de coordenadas (7) tiene la propiedad de ser canónico, lo que significa que el sistema (8) es también Hamiltoniano con Hamiltoniano $H=\omega I$.

\section{Oscilador armónico 2 dimensional}

El oscilador armónico 2-dimensional consiste de dos osciladores armónicos 1-dimensionales no acoplados, es decir, es el sistema de ecuaciones diferenciales de segundo orden en el plano $\mathbb{R}^{2}$ dado por

$$
\ddot{q}_{1}+\omega_{1} q_{1}=0, \quad \ddot{q}_{2}+\omega_{2} q_{2}=0, \quad\left(q_{1}, q_{2}\right) \in \mathbb{R}^{2}
$$

con $\omega_{1}$ y $\omega_{2}$ constantes positivas. De manera análoga al caso 1-dimensional, si introducimos las variables $p_{1}=\dot{q}_{1}, p_{2}=\dot{q}_{2}$ el sistema de ecuaciones (9) se transforma en el siguiente sistema de ecuaciones diferenciales lineales de primer orden en $\mathbb{R}^{4}$

$$
\begin{array}{ll}
\dot{q}_{1}=p_{1}, & \dot{q}_{2}=p_{2}, \\
\dot{p}_{1}=-\omega_{1}^{2} q_{1}, & \dot{p}_{2}=-\omega_{2}^{2} q_{2} .
\end{array}
$$

El oscilador armónico 2-dimensional en (10) resulta ser un sistema Hamiltoniano (con dos grados de libertad) con Hamiltoniano

$$
H\left(q_{1}, q_{2}, p_{1}, p_{2}\right)=\frac{1}{2}\left(p_{1}^{2}+\omega_{1}^{2} q_{1}^{2}\right)+\frac{1}{2}\left(p_{2}^{2}+\omega_{2}^{2} q_{2}^{2}\right) .
$$


El campo Hamiltoniano asociado al oscilador armónico 2-dimensional (10) está dado por

$$
X_{H}=\left(p_{1}, p_{2},-\omega_{1}^{2} q_{1},-\omega_{2}^{2} q_{2}\right)
$$

y su flujo por

$$
\mathrm{Fl}_{X_{H}}^{t}\left(\begin{array}{c}
q_{1} \\
q_{2} \\
p_{1} \\
p_{2}
\end{array}\right)=\left(\begin{array}{c}
q_{1} \cos \omega_{1} t+\frac{p_{1}}{\omega_{1}} \operatorname{sen} \omega_{1} t \\
q_{2} \cos \omega_{2} t+\frac{p_{2}}{\omega_{2}} \operatorname{sen} \omega_{2} t \\
-\omega_{1} q_{1} \operatorname{sen} \omega_{1} t+p_{1} \cos \omega_{1} t \\
-\omega_{2} q_{2} \operatorname{sen} \omega_{2} t+p_{2} \cos \omega_{2} t
\end{array}\right)
$$

A pesar de que el flujo del oscilador armónico 2-dimensional tiene componentes periódicas; su flujo no es periódico, en general. En efecto, el flujo del oscilador armónico (12) es periódico si y sólo si el cociente de las frecuencias $\omega_{1} / \omega_{2}$ es un número racional. En el caso general, es decir, sin importar que valor tomen las frecuencias $\omega_{1}$ y $\omega_{2}$; el oscilador armónico 2dimensional (10) posee dos familias de trayectorias periódicas. Una familia se caracteriza por tener periodo $T_{1}=2 \pi / \omega_{1}$ y cada miembro de esa familia contiene un punto de la forma $\left(E_{1}, 0,0,0\right)$ para algún numero real positivo $E_{1}$. La otra familia de trayectorias periódicas tiene período $T_{2}=2 \pi / \omega_{2}$ y cada trayectoria contiene un punto de la forma $\left(0, E_{2}, 0,0\right)$ para algún real positivo $E_{2}$. Estas familias de soluciones son especiales y usualmente son llamadas modos normales, [2].

Notemos que además del Hamiltoniano $H$ (11) las siguientes funciones

$$
\begin{aligned}
& f_{1}\left(q_{1}, q_{2}, p_{1}, p_{2}\right)=\frac{1}{2}\left(p_{1}^{2}+\omega_{1}^{2} q_{1}^{2}\right), \\
& f_{2}\left(q_{1}, q_{2}, p_{1}, p_{2}\right)=\frac{1}{2}\left(p_{2}^{2}+\omega_{1}^{2} q_{2}^{2}\right),
\end{aligned}
$$

también son integrales primeras del oscilador armónico. Es decir, tanto $f_{1}$ como $f_{2}$ son constantes a lo largo de las soluciones del oscilador armónico 2-dimensional dadas por (12). En efecto, si componemos $f_{i}$ con el flujo del campo Hamiltoniano $X_{H}$, obtenemos nuevamente la función $f_{i}$ :

$$
\begin{aligned}
f_{i} \circ \mathrm{Fl}_{X_{H}}^{t}\left(q_{1}, q_{2}, p_{1}, p_{2}\right) & =\frac{1}{2}\left(\left(q_{i} \cos \omega_{i} t+\frac{p_{i}}{\omega_{i}} \operatorname{sen} \omega_{i} t\right)^{2}+\omega_{i}^{2}\left(-\omega_{i} q_{1} \operatorname{sen} \omega_{1} t+p_{i} \cos \omega_{i} t\right)^{2}\right), \\
& =\frac{1}{2}\left(p_{i}^{2}+\omega_{i}^{2} q_{i}^{2}\right)=f_{i}\left(q_{1}, q_{2}, p_{2}, p_{2}\right), \quad \text { para } \quad i=1,2 .
\end{aligned}
$$

Las integrales primeras $f_{1}$ y $f_{2}$ del oscilador armónico 2-dimensional no son independientes del Hamiltoniano $H$ pues se verifica que $H=f_{1}+f_{2}$. Además, estas dos integrales son de particular interés pues los flujos de sus campos Hamiltonianos conmutan, es decir, para todo $t, s \in \mathbb{R}$ se cumple que $\mathrm{Fl}_{X_{f_{1}}}^{t} \circ \mathrm{Fl}_{X_{f_{2}}}^{s}=\mathrm{Fl}_{X_{f_{2}}}^{s} \circ \mathrm{Fl}_{X_{f_{1}}}^{t}$, (ver $\left.[1,2]\right)$.

Ahora, vamos a estudiar las trayectorias del oscilador armónico 2-dimensional en el espacio de configuraciones, es decir, las soluciones del sistema de ecuaciones de segundo orden (9) 
ó la proyección del flujo (12) sobre el plano $\left(q_{1}, q_{2}\right)$. Las soluciones $\left(q_{1}(t), q_{2}(t)\right)$ del oscilador armónico determinan curvas parametrizadas en el plano cuyas trazas son llamadas curvas de Lissajous [6].

Sea $\alpha(t)$ la solución particular del sistema de ecuaciones de segundo orden (9) con condiciones iniciales $\alpha(0)=\left(a_{1}, a_{2}\right)$ y $\dot{\alpha}(0)=\left(b_{1}, b_{2}\right)$. De $(12)$, se tiene que $\alpha(t)$ tiene la siguiente forma

$$
\alpha(t)=\left(q_{1}(t), q_{2}(t)\right)=\left(a_{1} \cos \omega_{1} t+\frac{b_{1}}{\omega_{1}} \operatorname{sen} \omega_{1} t, a_{2} \cos \omega_{2} t+\frac{b_{2}}{\omega_{2}} \operatorname{sen} \omega_{2} t\right),
$$

Como la función (11) es una integral primera, se sigue que $H(\alpha(t), \dot{\alpha}(t))=H\left(a_{1}, a_{2}, b_{1}, b_{2}\right)=$ $E$, donde $E$ es una constane positiva. De aquí se deduce que $\frac{1}{2}\left(\omega_{1}^{2} q_{1}^{2}(t)+\omega_{2}^{2} q_{2}^{2}(t)\right) \leq E$ para todo $t \in \mathbb{R}$. De esto tenemos que la solución $\alpha(t)$ está contenida en el interior de la elipse de ecuación

$$
\frac{q_{1}^{2}}{2 E / \omega_{1}^{2}}+\frac{q_{2}^{2}}{2 E / \omega_{2}^{2}}=1
$$

De hecho, vamos a mostrar que existe un rectángulo inscrito en la elipse que contiene en su interior a la solución particular $\alpha(t)$. Para esto consideremos las integrales primeras en (13). Supongamos que $f_{i}(\alpha(t), \dot{\alpha}(t))=f\left(a_{1}, a_{2}, b_{1}, b_{2}\right)=E_{i}$ para $i=1$, 2. Es decir, $\frac{1}{2}\left(\dot{q}_{i}^{2}(t)+\omega_{i}^{2} q_{i}^{2}(t)\right)=E_{i}$ para todo $t$ con $i=1,2$. De esto obtenemos que $\left|q_{i}(t)\right| \leq A_{i}, \forall t$, con $A_{i}=\frac{\sqrt{2 E_{i}}}{\omega_{i}}$. En consecuencia, la solución particular $\alpha(t)$ se encuentra dentro del rectángulo

$$
R=\left\{\left(q_{1}, q_{2}\right)|| q_{1}\left|\leq A_{1},\right| q_{2} \mid \leq A_{2}\right\}
$$

Como $E=E_{1}+E_{2}$, el rectángulo $R$ (16) esta contenido en la elipse (3) (ver figura 3). Cabe

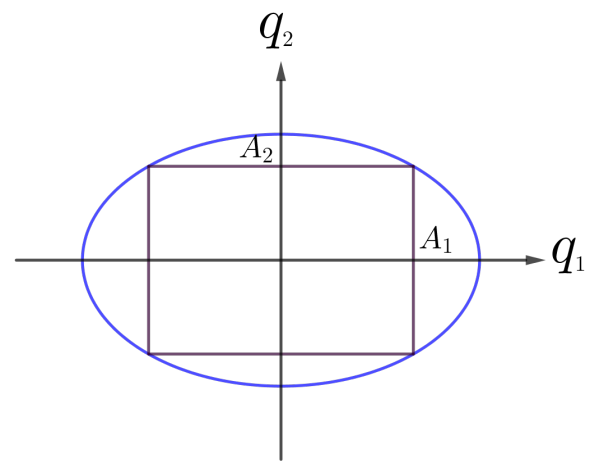

Figura 3: Rectángulo $R$ que contiene a la solución $\alpha(t)$

mencionar que la región $R$ depende tanto del valor de las frecuencias $\omega_{1}, \omega_{2}$ como de las condiciones iniciales de la solución particular $\alpha(t)$.

La cuestión ahora es tener una idea de cómo es la dinámica de la solución particular $\alpha(t)$ del sistema (9) dentro del rectángulo $R$ (16). Un análisis cualitativo muestra que las curva solución de (9) dada por (14) resultan ser altamente sensibles al cociente de las frecuencias $\frac{\omega_{1}}{\omega_{2}}$ y de las condiciones iniciales que se fijen para ésta solución. Por ejemplo, para el caso en el que $\frac{\omega_{1}}{\omega_{2}}=1$, las curvas de Lissaojus son elipses, incluyendo los casos particulares de 
círculos y segmentos de rectas, como se puede observar en las gráficas que aparecen en la figura 4.
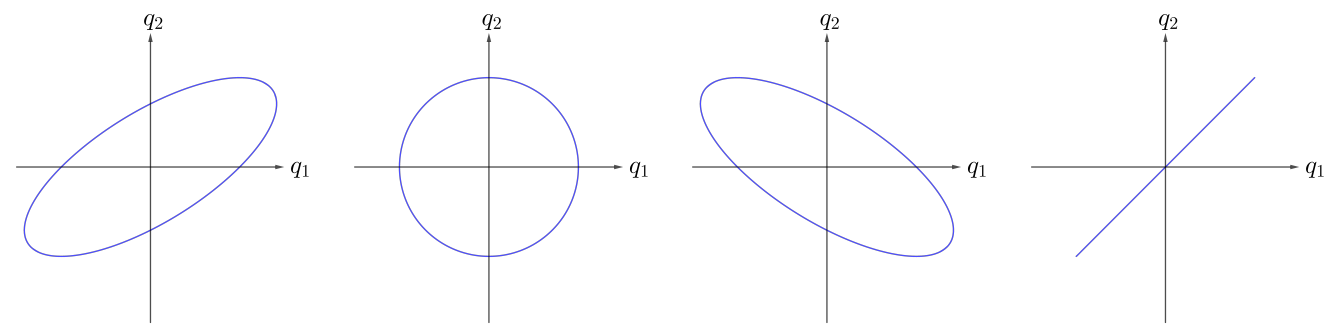

Figura 4: Curvas de Lissajous para $\omega_{1}=\omega_{2}$

En la figura 5 se muestran algunas soluciones del sistema (9) con coeficiente de frecuencias $\frac{\omega_{1}}{\omega_{2}}$ un número racional distinto de uno.
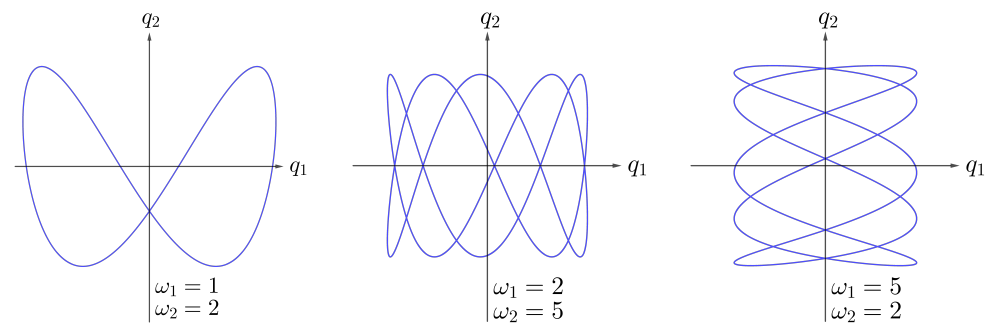

Figura 5: Caso $\frac{\omega_{1}}{\omega_{2}}$ racional

En el caso de que $\frac{\omega_{1}}{\omega_{2}}$ sea irracional la situación puede ser muy distinta. Si $\frac{\omega_{1}}{\omega_{2}}$ es irracional entonces las curvas solución del sistema (9) son densas en el rectángulo $R$ definido por (16), como se puede ver en la figura 6. Postergamos hasta la sección 6 la comprobación de este hecho (ver Observación 6.1).
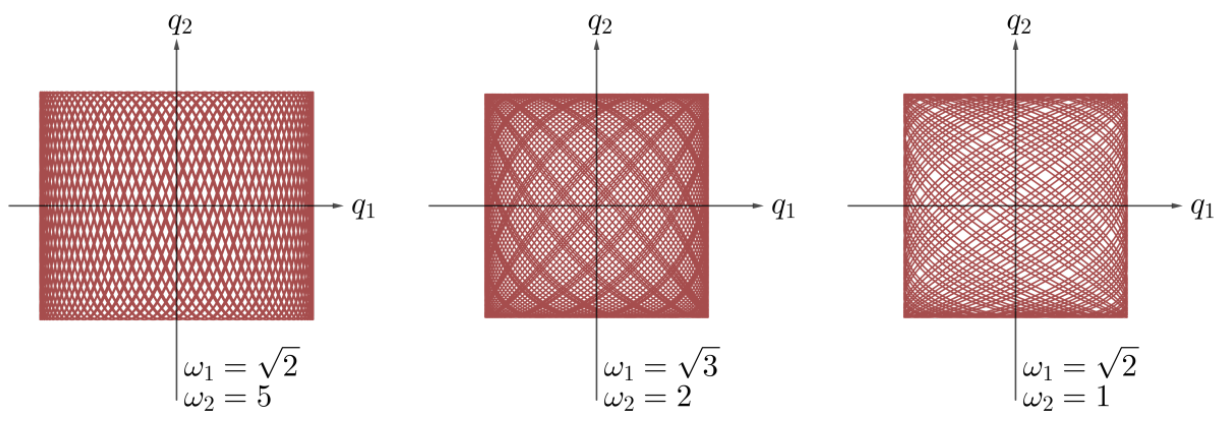

Figura 6: Caso $\frac{\omega_{1}}{\omega_{2}}$ irracional 
El oscilador como aproximación lineal. En varias aplicaciones, el oscilador armónico es sólo la aproximación a primer orden a algún problema físico. Por citar un ejemplo, consideremos una partícula de forma esférica con masa unitaria que se desliza sobre una superficie que se ubica sobre el plano $\left(q_{1}, q_{2}\right)$. Supongamos que la superficie tiene forma de un paraboloide elíptico con su mínimo en el origen, digamos que dicha superficie está dada por la gráfica de la función $f\left(q_{1}, q_{2}\right)=\frac{a_{1}}{2} q_{1}^{2}+\frac{a_{2}}{2} q_{2}^{2}$, con $a_{1}, a_{2}>0$ constantes. Despreciemos todos los posibles efectos de fricción y supongamos que la única fuerza que actúa sobre la partícula es la gravitatoria, con constante de graveda $g$. Independientemente de la velocidad inicial de la partícula, ésta se moverá en la dirección de máximo decrecimiento (el negativo del gradiente de la función $f(x, y))$. Al despreciar cualquier efecto de fricción, este movimiento continuará indefinidamente, aunque en la práctica la fricción entre la superficie y la partícula logrará que eventualmente la partícula alcance la posición de equilibrio en el mínimo de la función. Como la partícula solo puede moverse sobre la superficie, si queremos describir la trayectoria de la partícula, es suficiente con conocer la proyección de la trayectoria sobre el plano $\left(q_{1}, q_{2}\right)$, (ver figura 7 ). La dinámica que describe el movimiento de la trayectoria sobre le plano $\left(q_{1}, q_{2}\right)$ está determinada por el sistema de ecuaciones diferenciales de segundo orden no lineal

$$
\ddot{q}_{1}=\frac{-g a_{1} q_{1}}{1+a_{1}^{2} q_{1}^{2}+a_{2}^{2} q_{2}^{2}}, \quad \ddot{q}_{2}=\frac{-g a_{2} q_{2}}{1+a_{1}^{2} q_{1}^{2}+a_{2}^{2} q_{2}^{2}},
$$

como se puede verificar después de algunos cálculo elementales. La dinámica linealizada

— Trayectoria de la partícula sobre la superficie

- - - Proyección de la trayectoria sobre el plano $\left(q_{1}, q_{2}\right)$

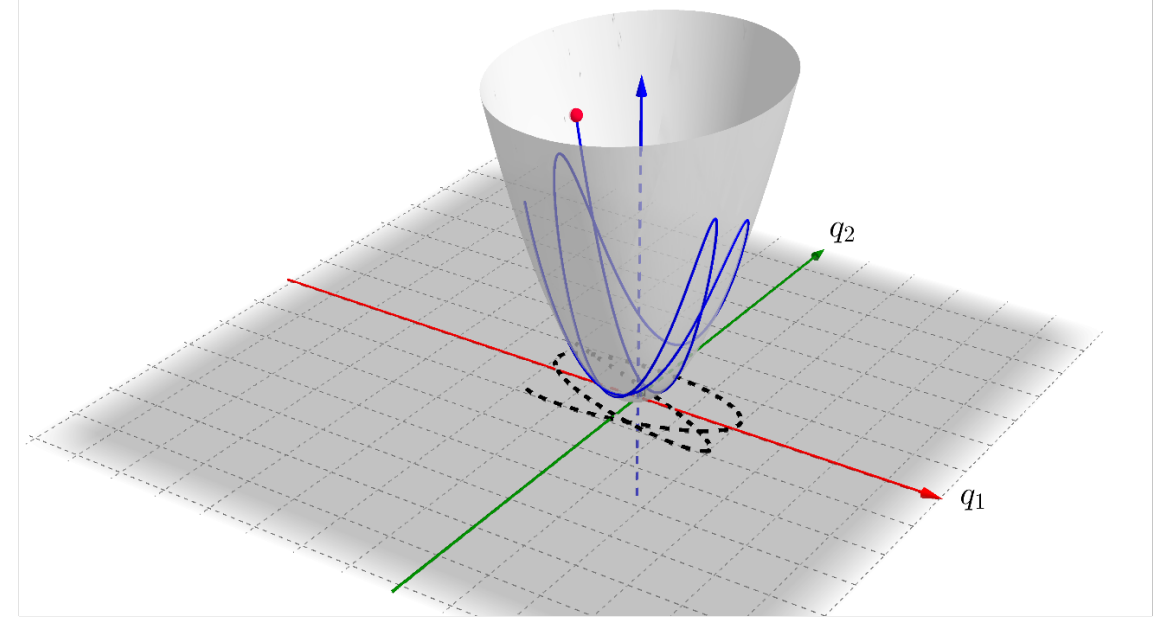

Figura 7: Partícula esférica deslizándose en un paraboloide elíptico

alrededor del origen del sistema (17) es justamente el oscilador armónico 2-dimensional (9), con frecuencias $\omega_{1}=\sqrt{g a_{1}}$ y $\omega_{2}=\sqrt{g a_{2}}$. Por ello, este sistema nos puede ayudar para determinar el comportamiento cualitativo de la dinámica del sistema alrededor del origen. 


\section{Geometría del oscilador armónico 2-dimensional: Toros de Liouville e inte- grabilidad de Liouville.}

En esta sección vamos a estudiar la geométrica detrás del oscilador armónico 2-dimensional. Consideremos la función $F: \mathbb{R}^{4} \rightarrow \mathbb{R}^{2}$ definida por las integrales primeras del oscilador armónico (13)

$$
\begin{aligned}
F(q, p) & =\left(f_{1}(q, p), f_{2}(q, p)\right) \\
& =\left(\frac{1}{2}\left(p_{1}^{2}+\omega_{1}^{2} q_{1}^{2}\right), \frac{1}{2}\left(p_{2}^{2}+\omega_{2}^{2} q_{2}^{2}\right)\right) .
\end{aligned}
$$

Como $f_{1}$ y $f_{2}$ son integrales primeras del oscilador armónico, las curvas solución de este sistema dadas por (12) están contenidos en los conjuntos de nivel de la función $F$. Es decir, los conjuntos de nivel de $F$ son conjuntos invariantes bajo el flujo del campo hamiltoniano $X_{H}$ del oscilador armónico 2-dimensional.

Describamos los conjuntos de nivel de la función $F(18)$ para determinar cómo son los conjuntos invariantes del oscilador armónico. Como $f_{1}, f_{2} \geq 0, F^{-1}\left(E_{1}, E_{2}\right) \neq \emptyset$ si y sólo si $E_{1}, E_{2} \geq 0$. Recordemos que $\left(E_{1}, E_{2}\right) \in \mathbb{R}^{2}$ es un valor regular de $F$ si para cada $(q, p) \in$ $F^{-1}\left(E_{1}, E_{2}\right)$, la matriz Jacobiana de $F$ tiene rango maximal en $(q, p)$. Por el Teorema de la Función Implícita [11], si $\left(E_{1}, E_{2}\right)$ es un valor regular de $F$ entonces el conjunto $F^{-1}\left(E_{1}, E_{2}\right)$ es una subvariedad dos dimensional en $\mathbb{R}^{4}$, es decir, un superficie. Si calculamos el Jacobiano de la función $F(18)$ en el punto $(q, p)$

$$
D F(q, p)=\left(\begin{array}{cccc}
\omega_{1}^{2} q_{1} & 0 & p_{1} & 0 \\
0 & \omega_{2}^{2} q_{2} & 0 & p_{2}
\end{array}\right)
$$

tenemos que la matriz tiene rango maximal en $\left(q_{1}, q_{2}, p_{1}, p_{2}\right)$ si y sólo si $\left(p_{1}, q_{1}\right) \neq 0$ y $\left(p_{2}, q_{2}\right) \neq 0$. Como el conjunto donde el rango es maximal es denso en $\mathbb{R}^{4}$, tenemos que casi todo el espacio fase del sistema está foliado por varidedades invariantes dos dimensionales. Más aún, los conjuntos de nivel que corresponden a valores regulares de $F$ son superficies difeomorfas a toros dos-dimensional inmersos en $\mathbb{R}^{4}$, como verificaremos a continuación. Fijemos una pareja de reales positivos $E_{1}, E_{2}$. Entonces

$$
\begin{aligned}
F^{-1}\left(E_{1}, E_{2}\right) & =\left\{\left(q_{1}, q_{2}, p_{1}, p_{2}\right) \in \mathbb{R}^{4} \mid F\left(q_{1}, q_{2}, p_{1}, p_{2}\right)=\left(E_{1}, E_{2}\right)\right\}, \\
& =\left\{\left(q_{1}, p_{1}\right) \in \mathbb{R}^{2} \mid \omega_{1}^{2} q_{1}^{2}+p_{1}^{2}=2 E_{1}\right\} \times\left\{\left(q_{2}, p_{2}\right) \in \mathbb{R}^{2} \mid \omega_{2}^{2} q_{2}^{2}+p_{2}^{2}=2 E_{2}\right\}, \\
& \cong \mathbb{S}^{1} \times \mathbb{S}^{1}=\mathbb{T}^{2} .
\end{aligned}
$$

Además, en caso de que $E_{1}$ ó $E_{2}$ sean cero, $F^{-1}\left(E_{1}, E_{2}\right)$ es una curva cerrada (trayectoria periódica de $X_{H}$ ) y si ambos son cero ésta pre-imagen consiste de un solo punto (el origen). En resumen, casi todo el espacio fase está foliado por toros invariantes dos dimensionales, llamados toros de Liouville, (ver [2, 4]). El resto del espacio está foliados por "hojas singulares", llamadas así porque, al ser curvas o un punto, tienen menor dimensión que los toros que las rodean. Dichas singularidades corresponden al caso en que alguno de los dos osciladores (o ambos) se encuentra en posición de equilibrio, $\left(E_{1}=0\right.$ ó $\left.E_{2}=0\right)$ y corresponden a los modos normales. 


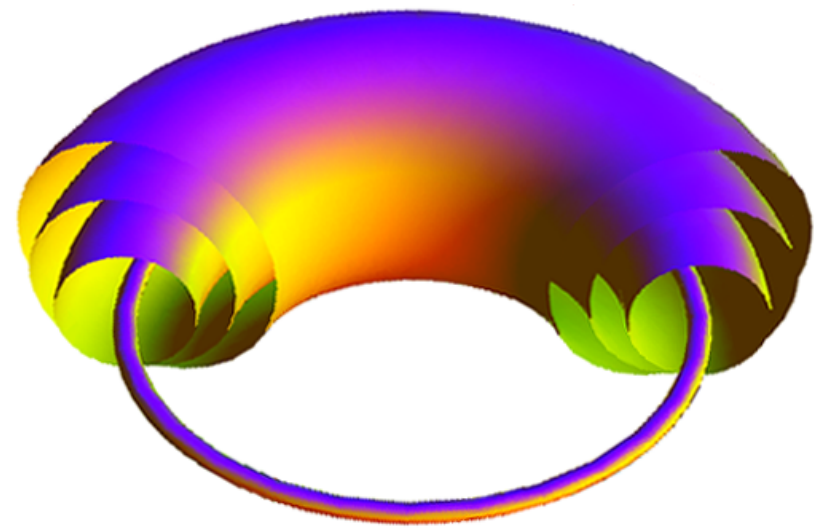

Figura 8: Foliación de Liouville por toros invariantes.

\section{Dinámica del oscilador armónico sobre los toros de Liouville}

Hasta este momento se ha demostrado que "casi"todas las trayectorias del oscilador armónico 2-dimensional están contenidas en toros dos dimensionales en $\mathbb{R}^{4}$. La cuestión ahora es determinar cómo es la dinámica del oscilador armónico sobre los toros de Liouville. Esto último depende de las propiedades aritméticas de las frecuencias $\omega_{1}, \omega_{2}$ y cualitativamente se tienen los siguientes casos:

(i) Si $\frac{\omega_{1}}{\omega_{2}}$ es racional, todas las trayectorias del oscilador armónico 2-dimensional son periódicas. Como los toros de Liouville son invariantes, éstos últimos deben estar foliados por dichas órbitas periódicas. Esta situación refleja la existencia de una tercer integral primera para el oscilador armónico la cual resulta ser funcionalmente independiente de $f_{1}$ y $f_{2}[3,4]$.

(ii) Si $\frac{\omega_{1}}{\omega_{2}}$ es irracional, no todas las trayectorias del oscilador armónico son periódicas. Precisamente, las órbitas que viven en los toros invariantes no lo son. Por lo tanto, cada trayectoria con punto inicial en un toro de Liouville se enrrolla sobre dicho toro pero nunca vuelve al punto inicial. De hecho, las trayectorias del oscilador llenan densamente los toros de Liouville.

Para estudiar el comportamiento cualitativo de las trayectorias del oscilador armónico 2-dimensional sobre los toros de Liouville y comprobar que, efectivamente, se presentan las situaciones descritas previamente, vamos a utilizar un cambio de coordendas que simplificará el análisis del sistema Hamiltoniano (10). Como en el caso 1-dimensional, vamos a introducir unas coordenadas especiales $\left(\varphi_{1}, \varphi_{2}, I_{1}, I_{2}\right)$ para el espacio fase $\mathbb{R}^{+} \times \mathbb{R}^{+} \times \mathbb{T}^{2}$, llamadas coordenadas acción-ángulo.

Notemos que el plano $q_{2}=p_{2}=0$ en $\mathbb{R}^{4}$ está foliado por órbitas periódicas del oscilador armónico 2-dimensional, a saber,los modos normales. Por tanto, esta familia de curvas satisface la condición $f_{2} \circ \mathrm{Fl}_{X_{H}}^{t}\left(q_{1}, 0, p_{1}, 0\right)=0$. Tomemos un número real positivo $E_{1}>0$ 
fijo y denotemos por $\mathcal{D}\left(E_{1}\right)$ al dominio en el plano $q_{2}=p_{2}=0$ acotado por la trayectoria $\gamma_{E_{1}}(t)=\mathrm{Fl}_{X_{H}}^{t}\left(\sqrt{2 E_{1} / \omega_{1}}, 0,0,0\right)$. Sea $A\left(E_{1}\right)=\oint_{\gamma_{E_{1}}(t)} p_{1} d q_{1}$, el área del dominio $\mathcal{D}\left(E_{1}\right)$. Entonces, el periodo $T\left(E_{1}\right)$ de la curva es dado por $T\left(E_{1}\right)=\frac{\partial A\left(E_{1}\right)}{\partial E_{1}}$. Definimos un par de coordenadas acción-ángulo por

$$
I_{1}=\frac{1}{2 \pi} A\left(E_{1}\right), \quad \varphi_{1}=\frac{2 \pi t}{T\left(E_{1}\right)}, \quad I_{1}>0, \quad \varphi_{1} \in \mathbb{S}^{1} .
$$

La misma situación ocurre sobre el plano $q_{1}=p_{1}=0$, por lo que podemos repetir los argumentos anteriores y definir el otro par de coordenadas acción-angulo por

$$
I_{2}=\frac{1}{2 \pi} A\left(E_{2}\right), \quad \varphi_{2}=\frac{2 \pi t}{T\left(E_{2}\right)}, \quad I_{2}>0, \quad \varphi_{2} \in \mathbb{S}^{1} .
$$

Usando las fórmulas (19), (20) y el flujo del oscilador armónico 2-dimensional (12) se construye el cambio de coordenadas $\Psi:\left(\varphi_{1}, \varphi_{2}, I_{1}, I_{2}\right) \mapsto\left(q_{1}, q_{2}, p_{1}, p_{2}\right)$ dado por

$$
q_{i}=\sqrt{\frac{2 I_{i}}{\omega_{i}}} \cos \left(\varphi_{i}\right), \quad p_{i}=-\sqrt{2 \omega_{i} I_{i}} \operatorname{sen}\left(\varphi_{i}\right), \quad i=1,2
$$

Un cálculo directo usando las fórmulas en (21) permite verificar que en coordenadas acciónángulo el Hamiltoniano $H(q, p)$ del oscilador armónico (11) toma la forma

$$
H\left(\varphi_{1}, \varphi_{2}, I_{1}, I_{2}\right)=\omega_{1} I_{1}+\omega_{2} I_{2} .
$$

Expresemos ahora el sistema Hamiltoniano del oscilador armónico (10) en coordenadas acción-ángulo. Derivando con respecto a $t$ en ambos lados del cambio de coordenadas (21), obtenemos

$$
\begin{aligned}
& \dot{q}_{i}=\sqrt{\frac{1}{2 \omega_{i} I_{i}}} \dot{I}_{i} \cos \left(\varphi_{i}\right)-\sqrt{\frac{2 I_{i}}{\omega_{i}}} \operatorname{sen}\left(\varphi_{i}\right) \dot{\varphi}_{i}, \\
& \dot{p}_{i}=-\sqrt{\frac{\omega_{i}}{2 I_{i}}} \dot{I}_{i} \operatorname{sen}\left(\varphi_{i}\right)-\sqrt{2 \omega_{i} I_{i}} \cos \left(\varphi_{i}\right) \dot{\varphi}_{i}
\end{aligned}
$$

De esto se deriva la siguiente identidad:

$$
-\dot{p}_{i} \operatorname{sen}\left(\varphi_{i}\right)+\omega_{i} \dot{q}_{i} \cos \left(\varphi_{i}\right)=\sqrt{\frac{\omega_{i}}{2 I_{i}}} \dot{I}_{i}
$$

Por otra parte, como $q_{i}(t), p_{i}(t)$ son soluciones del oscilador armónico, $\dot{p}_{i}=-\omega_{i}^{2} q_{i}$ y $\dot{q}_{i}=p_{i}$, sustituyendo en el lado derecho de la ecuación (23) y usando el cambio de coordenadas (21) llegamos a que $\dot{I}_{i}=0$. Si ahora sustituimos este último resultado en (22) y usando nuevamente $(21)$ se obtiene que $\dot{\varphi}_{i}=\omega_{i}$.

En resumen, el oscilador armónico (10) en coordenadas acción-ángulo toma la forma:

$$
\begin{array}{ll}
\dot{I}_{1}=0, & \dot{I}_{2}=0, \quad I_{1}, I_{2} \in \mathbb{R}^{+}, \\
\dot{\varphi}_{1}=\omega_{1}, & \dot{\varphi}_{2}=\omega_{2}, \quad\left(\varphi_{1}, \varphi_{2}\right) \in \mathbb{T}^{2} .
\end{array}
$$


Como se puede observar, el sistema (24)-(25) es integrable por cuadraturas y considerablemente más simple de resolver que el sistema (10). La solución al problema valor inicial $\left(E_{1}, E_{2}, \theta_{1}, \theta_{2}\right) \in \mathbb{R}^{+} \times \mathbb{R}^{+} \times \mathbb{T}^{2}$ en $t=0$ esta dada por

$$
\begin{aligned}
I_{i}(t) & =E_{i}, \\
\varphi_{i}(t) & =t \omega_{i}+\theta_{i} \bmod 2 \pi \text { para } i=1,2 .
\end{aligned}
$$

Geométricamente, el conjunto $I_{1}=E_{1}, I_{2}=E_{2}$, para un par de números positivos $\left(E_{1}, E_{2}\right)$ en $\mathbb{R}^{+} \times \mathbb{R}^{+} \times \mathbb{T}^{2}$ determina un toro invariante del oscilador armónico (10). Este toro está parametrizado por las coordenadas $\left(\varphi_{1}, \varphi_{2}\right)$. De $(27)$ se sigue que la dinámica del oscilador armónico 2-dimensional en el toro $I_{1}=E_{1}, I_{2}=E_{2}$ es lineal. Sin embargo, y como hemos comentado anteriormente, el comportamiento de las soluciones del oscilador armónico sobre el toro invariante es altamente sensible de la relación $\omega_{1} / \omega_{2}$ entre las frecuencias, como mostraremos a continuación.

Primero, fijemos un toro invariante, es decir, tomemos $I_{1}=E_{1}, I_{2}=E_{2}$. Consideremos el sistema de coordenadas $\left(\varphi_{1}, \varphi_{2}\right) \in \mathbb{R} / 2 \pi \mathbb{Z} \times \mathbb{R} / 2 \pi \mathbb{Z}$ para este toro. La dinámica del oscilador armómico sobre el toro $I_{1}=E_{1}, I_{2}=E_{2}$ está determinada por la ecuaciones (25). Es conveniente pensar a las variables $\left(\varphi_{1}, \varphi_{2}\right)$ como variables en un cuadrado de longitud $2 \pi$, donde se indentifican los lados opuestos $\varphi_{1}=0$ con $\varphi_{1}=2 \pi$ y $\varphi_{2}=0$ con $\varphi_{2}=2 \pi$ para construir el toro, (figura 9). De la ecuación (25), se sigue que el oscilador armónico induce en el cuadrado el siguiente sistema dinámico

$$
\frac{d \varphi_{2}}{d \varphi_{1}}=\frac{\omega_{2}}{\omega_{1}}
$$

Es claro que las soluciones de esta ecuación en el plano $\left(\varphi_{1}, \varphi_{2}\right)$ son líneas rectas de pendiente constante $\frac{\omega_{2}}{\omega_{1}}$. Cuando una solución alcanza el lado $\varphi_{1}=2 \pi$ (supongamos que cuando esto ocurre $\varphi_{2}=\alpha$ ) esta solución instantáneamente aparece en el lado $\varphi_{1}=0$ (en el punto con coordenada $\varphi_{2}=\alpha$ ), y entonces la solución continua sobre la recta con pendiente $\frac{\omega_{2}}{\omega_{1}}$. Lo misma situación ocurre cuando la solución alcanza el lado $\varphi_{2}=2 \pi$. En la figura 9 se ilustra la situación previamente descrita. Sea $C$ el círculo $\varphi_{1}=0$ en el toro $I_{1}=E_{1}, I_{2}=E_{2}$, es decir,

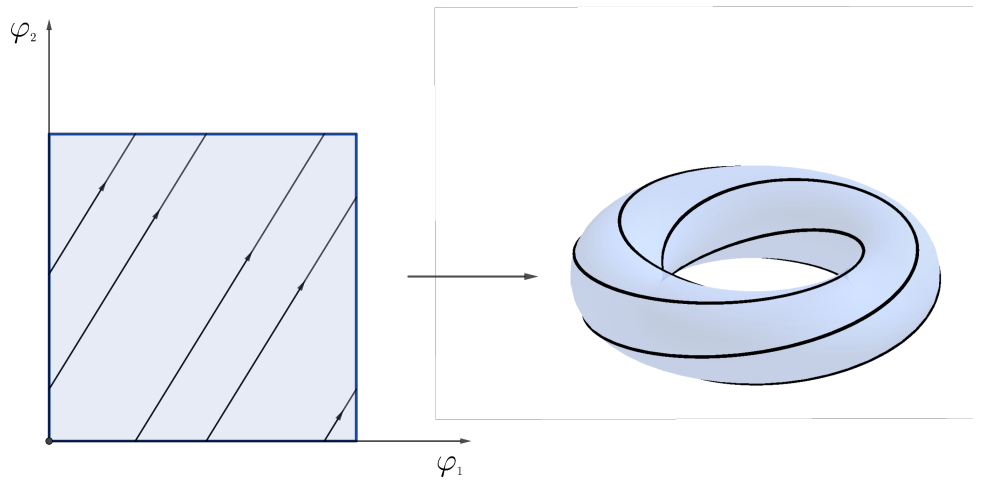

Figura 9: Dinámica del oscilador armonico sobre un toro invariante 
el lado izquierdo del cuadrado que representa nuestro toro. Si $\frac{\omega_{1}}{\omega_{2}}$ es un número racional, digamos $\frac{k}{l}$, entonces la solución de la ecuación (28) que inicie en un punto inicial $\left(\theta_{1}, \theta_{2}\right)$ en el toro (dentro del cuadrado de la figura 9), recorrerá $k$ veces de manera horizontal y $l$ veces de manera vertical antes de regresar al punto inicial. Estas son las soluciones periódicas que hemos comentado anteriormente. De aquí se concluye que la solución va a intersectar al círculo $C$ en un conjunto finito de puntos. Recíprocamente, si una solución en el toro corta al círculo $C$ en un conjunto finito de puntos entonces esa solución (y por lo tanto todas) tienen pendiente $\frac{\omega_{1}}{\omega_{2}}$ racional. Esto muestra que los toros de Liouville están foliados por órbitas periódicas del oscilador armónico.

Ahora, si $\frac{\omega_{1}}{\omega_{2}}$ es un número irracional entonces cada solución de la ecuación (28) intersectará al círculo $C$ en un número infinito de puntos. Vamos a mostrar que el conjunto de puntos de intersección es denso en el círculo $C$. De aquí se sigue fácilmente que las trayectorias del oscilador armónico 2-dimensional llenan densamente al toro invariante $I_{1}=E_{1}$, $I_{2}=E_{2}$ y por lo tanto a todos los toros de Liouville. Fijemos un punto en el círculo $C$ tomando $\varphi_{2}=\alpha$ para algún $0 \leq \alpha \leq 2 \pi$. Consideremos la solución de la ecuación (28) que pasa por $(0, \alpha)$. Cuando esta solución alcanza al círculo $\varphi_{1}=2 \pi$, ésta lo intersecta en el punto $\varphi_{2}=2 \pi \frac{\omega_{2}}{\omega_{1}}+\alpha \bmod 2 \pi$. Por nuestra identificación, la solución regresa al círculo $C$. Esto nos permite definir una función del círculo $C$ en sí mismo asignándole al punto $\alpha$ la coordenada $\varphi_{2}$ del punto en la solución de (28) cuando ésta regresa por primera vez al círculo. Explícitamente, tenemos la función

$$
\begin{aligned}
f & : \quad[0,2 \pi] \rightarrow[0,2 \pi], \\
f(\alpha) & =\alpha+\pi \frac{\omega_{2}}{\omega_{1}} \bmod 2 \pi .
\end{aligned}
$$

Notemos que $f$ es una isometría en el círculo $C$ (identificado con el intervalo $[0,2 \pi]$ ) si consideramos la distancia que induce la métrica euclideana de $\mathbb{R}$ en el espacio cociente $\mathbb{R} / 2 \pi \mathbb{Z}$. Geométricamente, la función $f$ en (29) sobre el círculo rota puntos por un ángulo $2 \pi \frac{\omega_{2}}{\omega_{1}}$. Tomemos un punto $\theta_{0}$ en $C$ y consideremos su órbita bajo la función $f$, es decir, el conjunto $\left\{\theta_{n} \mid \theta_{n}=f\left(\theta_{n-1}\right), n \in \mathbb{N}\right\}$. La órbita de $\theta_{0}$ es precisamente la intersección del círculo $C$ en el toro $I_{1}=E_{1}, I_{2}=E_{2}$ con la trayectoria del oscilador armónico que pasa por el punto $\left(0, \theta_{0}\right)$. De aquí se sigue que la órbita de $\theta_{0}$ es un conjunto infinito, pues si tuviera un conjunto finito de puntos la trayectoria del oscilador armónico que pasa por el punto $\left(\theta_{0}, 0\right)$ sería períodica y esto es una contradicción. Probemos que la órbita de $\theta_{0}$ es densa en $C$. Sea $\alpha$ un punto en el círculo $C$ y $\varepsilon>0$ arbitrarios. Probemos que existe un $\theta_{n}$ tal que $\left|\alpha-\theta_{n}\right|<\varepsilon$. Como la órbita de $\theta_{0}$ es infinita, existen enteros $k>l$ tales que $\left|\theta_{k}-\theta_{l}\right|<\varepsilon$ ya que el círculo tiene circunferencia finita. Como $f$ es una isometría, tenemos que $\left|\theta_{0}-\theta_{k-l}\right|<\varepsilon$. Ahora, como $\theta_{k-l}$ se obtiene rotando $\theta_{0}$ por un ángulo $(k-l) 2 \pi \frac{\omega_{2}}{\omega_{1}}$ que, módulo $2 \pi$, es un ángulo menor que $\varepsilon$. Realizando repetidamente una rotación por este ángulo, obtenemos una sucesión de punto en la órbita de $\theta_{0}$, definida por $\vartheta_{r}=\theta_{0}+r(k-l) 2 \pi \frac{\omega_{2}}{\omega_{1}} \bmod 2 \pi$. Esta sucesión tiene la propiedad de que $\left|\vartheta_{r}-\vartheta_{r-1}\right|<\varepsilon$ para todo natural $r$. Para $\alpha$ un punto en el círculo $C$, si este punto no pertenece a la sucesión de puntos $\vartheta_{n}$, entonces tomemos el entero $r$ tal que $\vartheta_{r}$ minimiza la distancia de $\alpha$ al conjunto $\left\{\vartheta_{n}\right\}$. Por la propiedad de este conjunto, se sigue que $\left|\vartheta_{r}-\alpha\right|<\varepsilon$ y por lo tanto la órbita es densa, como queríamos probar. 
Observaciones 6.1 Como corolario de lo anteriormente expuesto y usando el cambio de coordenadas (21) se sigue fácilmente que las curvas solución del sistema (9) son densas en el ractángulo $R(16)$ cuando $\frac{\omega_{1}}{\omega_{2}}$ es irracional.

Visualización de la trayectorias en los toros de Liouville. Para concluir esta sección, vamos presentar unas imágenes que ilustran la dinámica del oscilador armónico 2-dimensional sobre los toros de Liouville descrita previamente. Formalmente, los toros de Liouville son subconjunto conjuntos de $\mathbb{R}^{4}$, por lo que no podemos tener un representación visual de estos como subconjuntos del espacio fase. Sin embargo, podemos sacar provecho que los toros son superficies 2-dimensionales y que éstas son superficies que se pueden encajar en $\mathbb{R}^{3}$. Para esto, haremos uso de la siguiente parametrización del toro en $\mathbb{R}^{3}$ dada por

$$
\begin{aligned}
\psi & :[0,2 \pi,] \times[0,2 \pi] \rightarrow \mathbb{R}^{3}, \\
\psi\left(\varphi_{1}, \varphi_{2}\right) & =\left(\left(R+r \cos \varphi_{1}\right) \cos \varphi_{2},\left(R+r \cos \varphi_{1}\right) \operatorname{sen} \varphi_{2}, r \operatorname{sen} \varphi_{2}\right),
\end{aligned}
$$

con $R>r>0$ números reales. La imagen de $[0,2 \pi] \times[0,2 \pi]$ bajo la parametrización $\psi$ es un toro en $\mathbb{R}^{3}$ que se construye precisamente "pegando"los lados del cuadrado, tal y como se ilustra en la figura 9 .

Para trazar una curva solución del oscilador armónico 2-dimensional sobre un toro, debemos fijar un par de valores para $R$ y $r$ para la parametrización $\psi$ en (30). Esto es equivalente a fijar las coordenadas $I_{1}=E_{1}, I_{2}=E_{2}$ en $\left(I_{1}, I_{2}, \varphi_{1}, \varphi_{2}\right) \in \mathbb{R}^{+} \times \mathbb{R}^{+} \times \mathbb{T}^{2}$ y que hemos identificado con el cuadrado $[0,2 \pi] \times[0,2 \pi]$, pues en coordenadas acción-ángulo la dinámica oscilador armónico 2-dimensional con Hamiltoniano $H\left(I_{1}, I_{2}, \varphi_{1}, \varphi_{2}\right)=\omega_{1} I_{1}+\omega_{2} I_{2}$ está determinada por el sistema (24), (25). De (27), se tiene que la solución particular $\gamma(t)=\left(\varphi_{1}(t), \varphi_{2}(t)\right)$ del oscilador armónico 2-dimensional sobre el toro $[0,2 \pi] \times[0,2 \pi]$ tal que $\gamma(0)=\left(\theta_{1}, \theta_{2}\right)$ está dada por

$$
\begin{aligned}
\gamma & : \mathbb{R} \rightarrow[0,2 \pi] \times[0,2 \pi], \\
\gamma(t) & =\left(\omega_{1} t+\theta_{1} \bmod 2 \pi, \omega_{2} t+\theta_{2} \bmod 2 \pi\right) .
\end{aligned}
$$

Combinando la solución particular $\gamma(t)$ con la parametrización (30), tenemos que la curva

$$
\begin{aligned}
& \psi(t):=\psi(\gamma(t))=\left(\left(R+r \cos \left(\omega_{1} t+\theta_{1}\right)\right) \cos \left(\omega_{2} t+\theta_{2}\right),\right. \\
&\left.\left(R+r \cos \left(\omega_{1} t+\theta_{1}\right)\right) \operatorname{sen}\left(\omega_{2} t+\theta_{2}\right), r \operatorname{sen}\left(\omega_{2} t+\theta_{2}\right)\right),
\end{aligned}
$$

representa una trayectoria en del oscilador armónico sobre un toro en $\mathbb{R}^{3}$ que sí podemos visualizar. Las imágenes que se presentan a continuación para ilustrar la dinámica del oscilador sobre los toros de Liouville han sido construidas usando precisamente la curva (32).

Caso racional. La figura 10 contiene varias imágenes que corresponden a trayectorias del oscilador armónico con frecuencias $\omega_{1}=1 \mathrm{y} \omega_{2}=2$. En la figura 10a solo aparece una curva pues queremos resaltar el hecho que en el caso racional las curvas solución son periódicas (curvas cerradas). En las figuras 10b, 10c y 10d aumentamos el número de curvas solución para visualizar cómo el toro está foliados por las órbitas periódicas del oscilador armónico. 


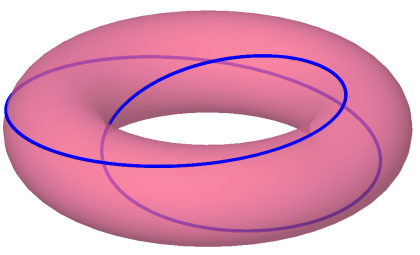

(a) Una curva solución

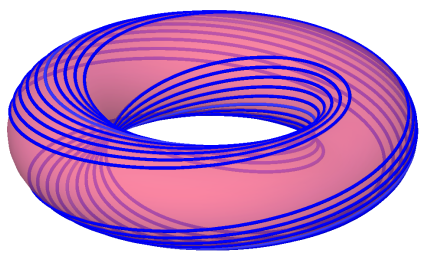

(c) Nueve curvas solución

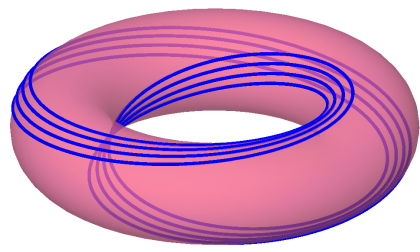

(b) Cuatro curvas solución

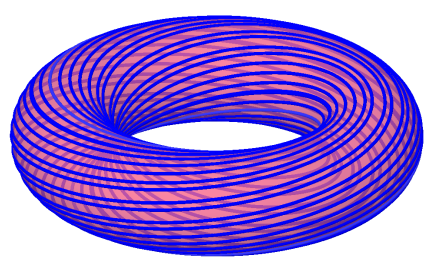

(d) Diecisiete curvas solución

Figura 10: Trayectorias del oscilador sobre el toro. $\frac{\omega_{1}}{\omega_{2}}$ racional

Caso irracional. Para ilustrar el caso irracional, se tomaron los valores de frecuencias $\omega_{1}=5$ y $\omega_{2}=\pi$. Como en este caso las trayectorias son densas en los toros de Liouville, es suficiente trazar una curva solución del oscilador armónico usando $\psi(t)$ dada por (32) para algún valor inicial y dejar el tiempo correr para ver como ésta llena al toro. En la figura 11, se muestra en varias imágenes como evoluciona una órbita del oscilador sobre el toro. En cada imagen aparece la traza de la misma trayectoria del oscilador pero en distintos intervalos de tiempo. En las primeras imágenes, que corresponden a intervalos de tiempo más pequeños, se puede apreciar que las trayectorias no son cerradas y al parecer su comportamiento es un poco caótico. Conforme aumentamos el intervalo de tiempo podemos ver como la trayectoria se enrolla sobre el toro cubriendo cada más puntos de éste hasta la última imagen en la que ya no es posible distinguir los puntos sobre el toro por donde no pasa la trayectoria. 


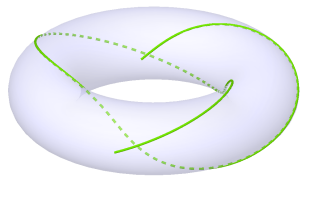

$0 \leq t \leq \pi$

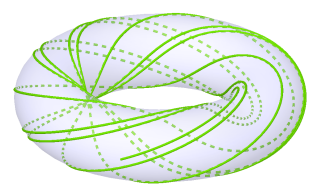

$0 \leq t \leq 5 \pi$

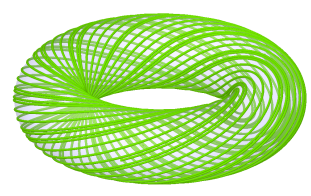

$0 \leq t \leq 20 \pi$

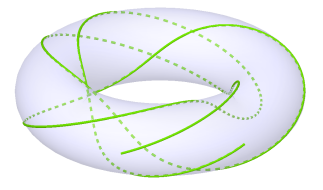

$0 \leq t \leq 2 \pi$

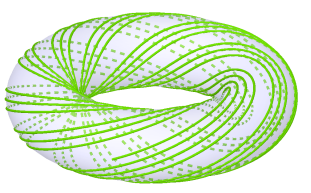

$0 \leq t \leq 10 \pi$

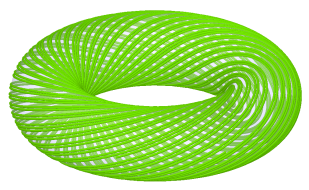

$0 \leq t \leq 30 \pi$

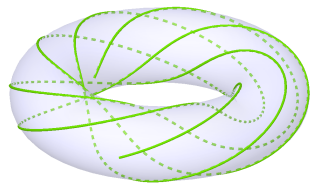

$0 \leq t \leq 3 \pi$

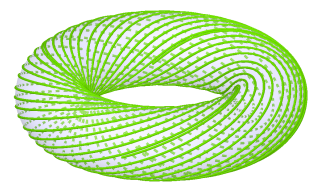

$0 \leq t \leq 15 \pi$

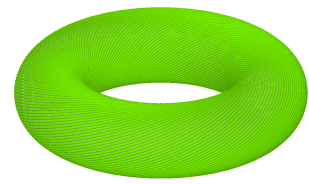

$0 \leq t \leq 50 \pi$

Figura 11: Trayectoria del oscilador sobre el toro. $\frac{\omega_{1}}{\omega_{2}}$ irracional

Agradecimientos. El autor de este trabajo desea expresar su profundo agradecimiento a los evaluadores de este trabajo por su oportunas críticas, comentarios y sugerencias que ayudaron a mejorarlo de forma importante.

\section{Referencias}

[1] R. Abraham and J. E. Marsden, Foundations of Mechanics, AMS Chelsea Publishing, 2nd Edition, (1978).

[2] V. I. Arnold, Mathematical Methods of Classical Mechanics, Vol. 60 of GTM, SpringerVerlag, 2nd edition, (1989).

[3] R. Cushman and L. Bates, Global Aspects of Classical Integrable Systems, BirkhäuserVerlag, Berlin, (1997).

[4] F. Fassò, Superintegrable Hamiltoninan Systems: Geometry and Perturbations, Acta Appl. Math., Vol 87, pp. 93-121 (2005) 
[5] H. Goldstein, C. Poole and J. Safko, Classical Mechanics, Addison-Wesley, Third ed., (2002).

[6] T. B. Greenslade, Adventures with Lissajous Figures, Morgan \& Claypool Publishers, (2018).

[7] B. C. Hall, Quantum Theory for Mathematicians, Vol 267 of GTM, Springer-Verlag, New York, (2013).

[8] M. W. Hirsch, S. Smale and R. L. Devaney, Differential Equations, Dynamical Systems and an Introduction to Chaos, Vol. 60 of Pure and Applied Mathematics, Elsevier, 2nd edition, (2004).

[9] J. Marsden and T. Ratiu, Introduction to Mechanics and Symmetry, Springer-Verlag, New York, (1994).

[10] K. R. Meyer, The Geometry of Harmonic Oscillators, The Amer. Math. Monthly,Vol. 97, no. 6, pp. 457-465 (1990).

[11] M. Spivak, Cálculo en Variedades, Editorial Reverté, (1988). 